\title{
MEMS Based Acoustic Energy Harvester
}

\author{
S.B.Rudraswamy ${ }^{1}$, Aditya D $S^{2}$, Harish G $V^{3}$, Pavan Kishore $M^{4}$, Vinay $V^{5}$ \\ ${ }^{1}$ Assistant Professor, Department of ECE, SJCE, Mysuru-570006 \\ 2,3,4,5 Department of ECE, SJCE, Mysuru-570006
}

\begin{abstract}
There is an enormous and ever quenching need for energy. Sound is available everywhere in different types (in different domains) and can be harnessed. The principle objective of this project is to design and simulate an electromechanical acoustic resonator for reclamation of energy using microelectro mechanical systems (MEMS) technology. Acoustic to mechanical transduction is achieved using a Helmholtz resonator, and mechanical to electrical transduction is accomplished using piezoelectric strip. The model with a cylindrical Helmholtz resonator and a Lead Zirconate Titanate (PZT) circular plate resonator was designed and analyzed using COMSOL MULTIPHYSICS 4.3a. In this study, a Helmholtz Resonator is used to collect travelling acoustic waves at frequencies of 4.5 $\mathrm{kHz}$ to $5.5 \mathrm{kHz}$. At the resonance of the Helmholtz Resonator, amplified resonant acoustic standing waves are developed inside the cavity. The pressure difference between the walls drive the vibration motion of the silicon membrane back plate, to which piezoelectric ring made of Lead Zirconate Titanate (PZT). The deformation induced as a result of this, leads to generate an electrical power via the direct piezoelectric effect.
\end{abstract}

Keywords: MEMS, Acoustic, Energy, harvesting

\section{Introduction}

The principal objective of our project is to convert acoustic energy into electrical energy by scavenging the vibrations of piezoelectric material. The diaphragm transduces an acoustical pressure fluctuation into a mechanical deformation, while the piezoelectric material transduces that mechanical deformation into an electrical signal (charge or voltage). The electrical energy can then either be stored or used directly for a particular circuit application. The relatively small geometries possible in MEMS make such a device useful for small, portable devices, where battery requirements are often difficult to meet. Additionally, the device is well suited to applications where wiring is difficult such as remote sensors or unwieldy as in the case of large arrays of devices requiring power.

\section{Methodology}

\section{Schematic}

The overall concept behind the energy harvester is illustrated in Figure 2. The main idea is to convert acoustic energy into a usable form of electrical energy. The figure illustrates a plane wave tube as the source of acoustic power. Some of the incident acoustic power is reflected at the end of the tube. However, a portion is transmitted through the energy harvester. The energy harvesterperforms the actual transduction of energy from the acoustical to the electrical energy domains. The electrical energy that exits the energy harvester has the same form as the input acoustic signal, which we assume for now is sinusoidal. The sinusoidal signal is then routed to the energy harvesting circuitry that serves to rectify and conditionthe signal before sending it on to the end application. In the example illustrated in Figure 2, the end application is a battery that is charged by the output of the harvesting circuitry. For the particular application considered, the initial energy is in the form of acoustical energy. In order to convert acoustical energy into electrical energy, a diaphragm based transducer is utilized. The diaphragm transducer utilizes the mechanical energy domain as an intermediary to transfer energy.

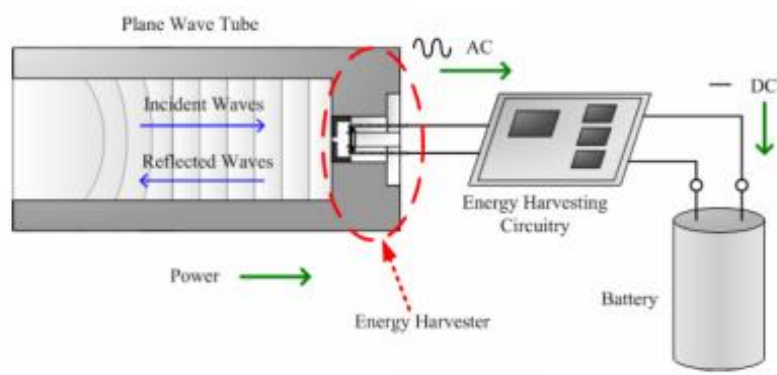

Figure 1: Schematic of overall energy harvesting concept

\section{Transduction}

The base of the energy harvester contains a piezoelectric ring. When excited by an acoustic input, a single resonance is seen, whereby the acoustic pressure inside the cavity is amplified to a level much greater than the incident acoustic signal. The Helmholtz resonator is very similar to an LCR resonant circuit. Both systems have a single degree-offreedom, with a single resonant peak, whereby the amplitude of the forcing function is greatly amplified.

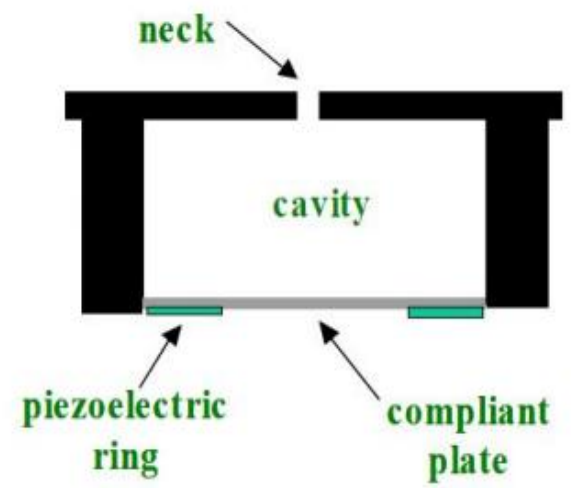

Figure 2: Transduction Arrangement 


\section{International Journal of Science and Research (IJSR) \\ ISSN (Online): 2319-7064}

Index Copernicus Value (2013): 6.14 | Impact Factor (2014): 5.611

\section{Design}

\section{Design of Helmholtz Resonator}

Rectangular comb drives find use in a variety of MEMS applications. The following model is an electrostatically actuated comb drive. The model includes just a few of the teeth. It simulates only the comb drive and its attachment using double-folded beam springs. The upper half of the comb is fixed, as is the end of the beam spring. The system applies an electric potential to the beam spring and the lower comb; the upper comb is grounded.

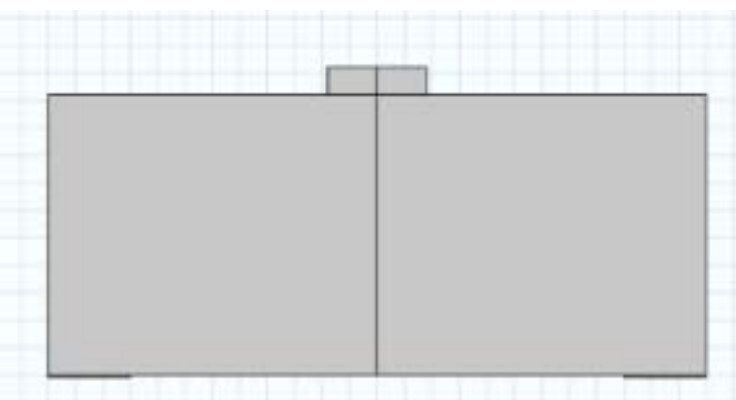

Figure 3: Dimensions of the Helmholtz Resonator

Table 1. Dimensions of the teeth of the comb drive actuator

- Cavity radius : $1200 \mu \mathrm{m}$

- Cavity height : $1020 \mu \mathrm{m}$

- Mouth radius : $180 \mu \mathrm{m}$

- Mouth height : $100 \mu \mathrm{m}$

- Piezoelectric strip thickness : $8 \mu \mathrm{m}$

- Piezoelectric outer radius : $1200 \mu \mathrm{m}$ (cavity radius)

- Piezoelectric inner radius : $900 \mu \mathrm{m}$

\section{Mathematical Equations}

Resonant frequency of the resonator is given by

$$
f_{H}=\frac{v}{2 \pi} \sqrt{\frac{A}{V_{o} L_{e q}}}
$$

Where, $\mathrm{V}$ is the speed of sound in gas A is cross sectional area of neck $V_{o}$ is static volume of the cavity $L_{e q}$ is equivalent length of the neck. Speed of sound in gas is given by,

$$
v=\sqrt{\frac{r P_{o}}{\rho}} .
$$

\section{Results}

After successfully designing the MEMS model the simulation results were obtained at the resonant frequency of $5.3 \mathrm{kHz}$.

\section{Voltage Plot}

Maximum voltage attained: $1.4 \mathrm{mV}$ (open circuit), $0.7 \mathrm{mV}$ at $5.3 \mathrm{kHz}$ with a load of $50 \Omega$.

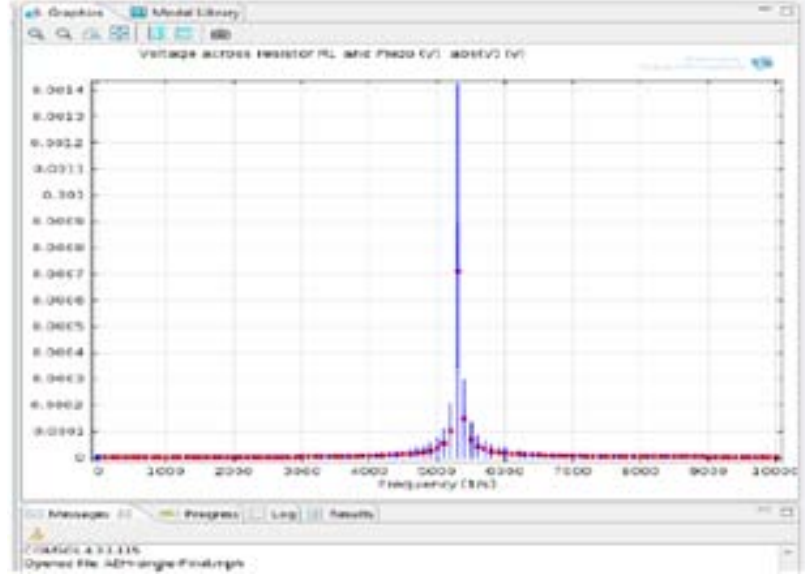

Figure 4: Voltage for Single Harvester

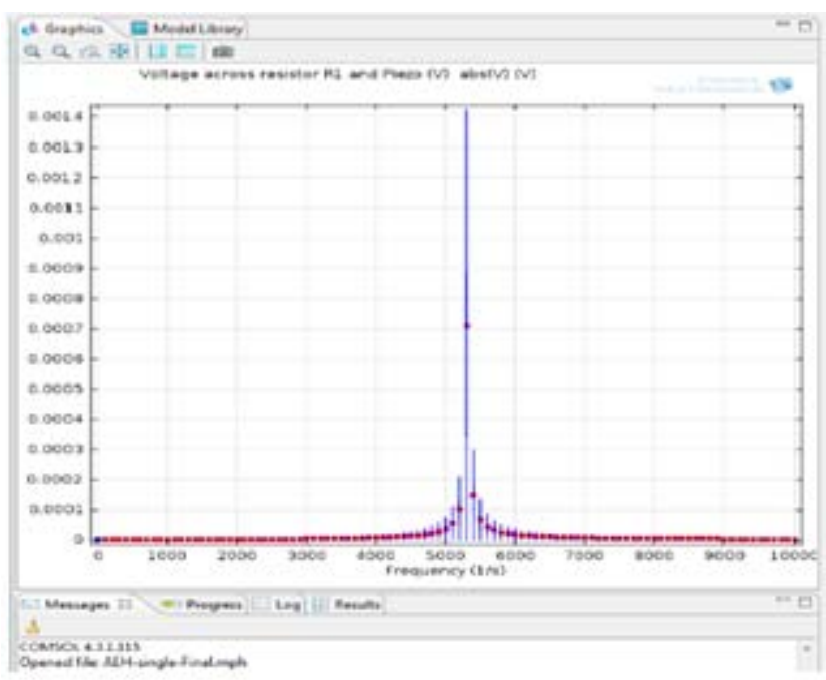

Figure 5: Voltage for Array Harvester

\section{Current Plot}

Maximum current attained: $14.2 \mathrm{~mA}$ at $5.3 \mathrm{kHz}$ with a load of $50 \mathrm{ohm}$

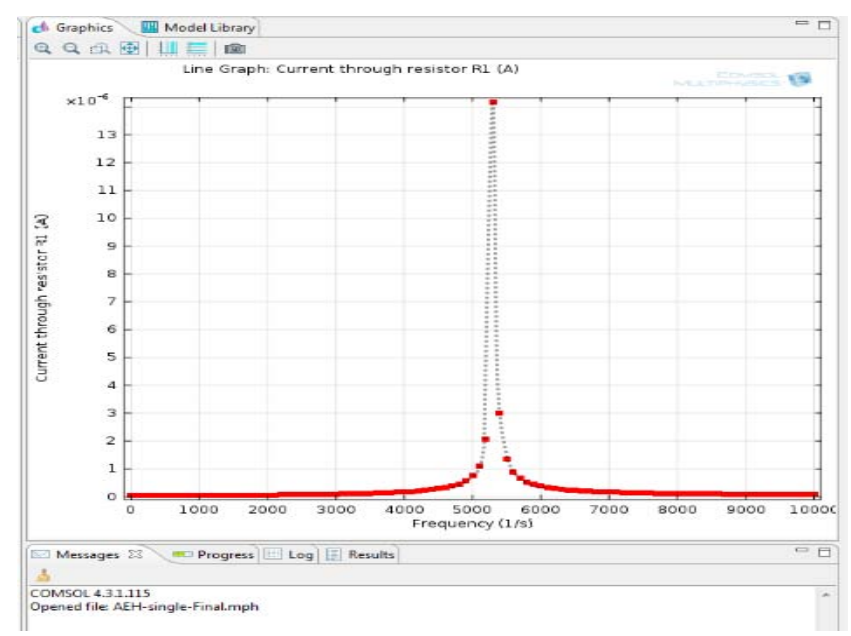

Figure 6: Current for Single Harvester 


\section{International Journal of Science and Research (IJSR) \\ ISSN (Online): 2319-7064}

Index Copernicus Value (2013): 6.14 | Impact Factor (2014): 5.611

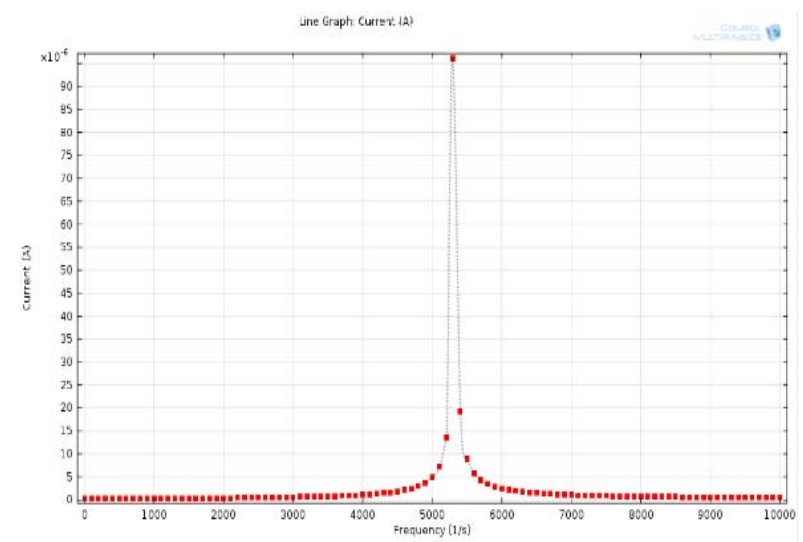

Figure 7: Current for Array Harvester

\section{Power Plot}

Maximum power dissipated: $10.06 \mathrm{nW}$ at $5.3 \mathrm{kHz}$ with a load of $50 \mathrm{ohm}$

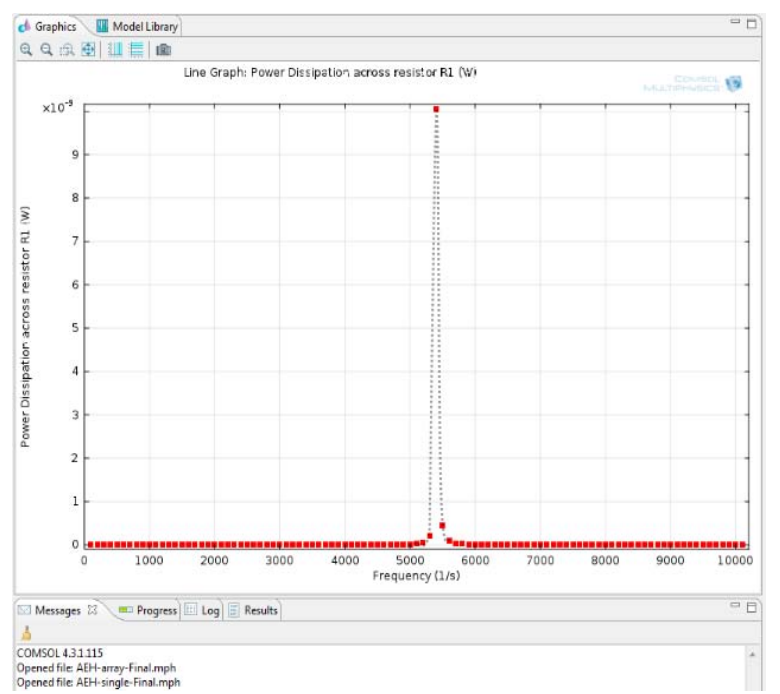

Figure 8: Power for Single Harvester

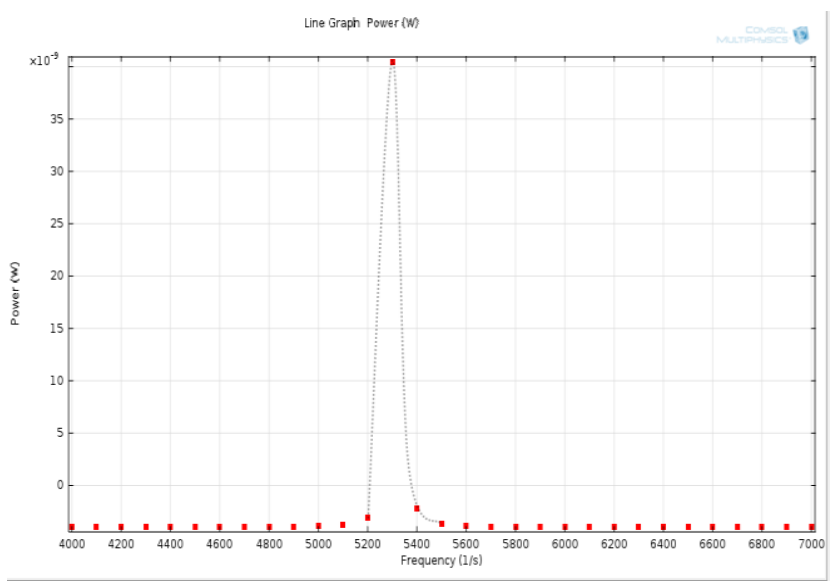

Figure 9: Power for Array Harvester

\section{Conclusion}

The idea of acoustic energy harvesting using a Helmholtz resonator is not new, many researches have already been made and they have obtained appropriate results. The prime contributor to the improvement that is evident in this work is replacement of conventional piezoelectric back plate with piezoelectric ring.
The piezoelectric back plate which was used in other works restricted the movement of the silicone diaphragm. It was loading the membrane and thus damped the vibrations. Thus it resulted in lesser deformation of the piezoelectric structure which is the basis for energy transduction, also maximum voltage variation was found at the centre. Using a ring, eliminates the above constraints. The ring is present at the circumference of the cavity and hence hardly loads the diaphragm. Thus it leads to more vibrations being transferred to the piezoelectric ring. Also the voltage variation occurs on the inner and outer radial surfaces, with the bottom surface being the ground. Thus harvesting is easy.

Furthermore, in simulation, with the incident wave of 100 $\mathrm{dB}$, the PZT ring has generated the maximum voltage of, 1.4 $\mathrm{mV}$ (at $5.3 \mathrm{kHz}$ ). By applying the resistance load of $50 \Omega$, the maximum power generated was found $10.3 * 10^{-9} \mathrm{~W}$. The approximate Q factor was found to be 53.This magnitude of power is more suitable comparing with the recent studies.

\section{References}

[1] Zurkinden A., Campanile F., et al, Wave Energy Converters through piezoelectric Polymers, Proceedings of the COMSOL Users Conference (Grenoble).

[2] Kamel T., Elfrink R, et al, Modeling and Characterization of MEMS based Piezoelectric Devices.

[3] www.academia.edu 